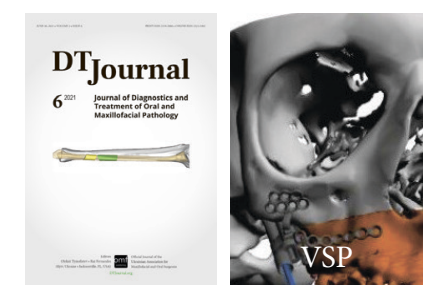

\title{
CASE
}

\section{Alternative Management of Severe Maxillary Asymmetry with Multi-vector Osteogenic Distraction and Customized Polyetheretherketone (PEEK) Prosthesis}

Hernán Arango , James Vidal ${ }^{b}$ Jorge Andres Moncadab , \& Juan Pablo Lópeze, *

\section{SUMMARY}

Osteogenic distraction has gotten an excellent value as a treatment of severe asymmetries. This report aims to present a treatment option to manage severe midface asymmetries using multi-vector devices and virtual planning for facial bone distraction and fixation with a customized polyetheretherketone (PEEK) prosthesis in a 16-year-old patient who at ten months of age was diagnosed and treated with chemotherapy and radiotherapy for embryonal rhabdomyosarcoma in the right orbit.

\footnotetext{
a Oral and Maxillofacial Surgeon, Professor, Universidad El Bosque, Bogotá, Colombia.

Oral and Maxillofacial Surgeon, Fundación Hospital Universitario Metropolitano, Barranquilla, Colombia.

${ }^{\text {b }}$ Oral and Maxillofacial Surgeon, Universidad El Bosque, Bogotá, Colombia.

c Year 2 Oral and Maxillofacial Surgery Resident, 4-year Residency Program, Universidad El Bosque.

Oral and Maxillofacial researcher, Fundacion Santa Fe de Bogotá, Bogotá, Colombia

* Corresponding author's address: Dr. Juan Pablo López,

Calle (Street) 129 \#7d-47, Bogotá, Colombia.

E-mail: jplopez2304@gmail.com

Phone: (57) 3136905010
}

Please cite this article as: Arango H, Vidal J, Moncada JA, López JP. Alternative management of severe maxillary asymmetry with multi-vector osteogenic distraction and customized polyetheretherketone (PEEK) prosthesis. J Diagn Treat Oral Maxillofac Pathol 2021;5(6):69-76.

The letters 'VSP' at the upper right icon means that article contains virtual surgical planning

Paper received 19 May 2021

Accepted 05 June 2021

Available online 05 June 2021

https://dx.doi.org/10.23999/j.dtomp.2021.6.2

(c) 2021 OMF Publishing, LLC. This is an open access article under the CC BY license (http://creativecommons.org/licenses/by-nc/4.0/). 


\section{INTRODUCTION}

Snyder et al began in 1972 a study on the craniofacial region about mandibular enlargement using bone distraction in dogs based on Abbott and Coleman's principles. ${ }^{1}$ Although at that time, there were only animal studies. McCarthy et al reported the first case series in children between 23 and 131 months old diagnosed with hemifacial microsomia and Nager's syndrome getting a success rate between 18 and 24 $\mathrm{mm}$ with an expansion dispositive. ${ }^{2}$ Rachmiel et al performed a midface advancement for the first time through gradual distraction on sheep, and their results were a mean of $33 \mathrm{~mm}$ of advanced without bone grafts. ${ }^{3}$ Polley et al published in 1995 a case report about craniofacial deformities associated with Pfeifer's syndrome in a child with midface deficiency; they managed it through osteogenic distraction and achieved a mandibular advancement around $35 \mathrm{~mm}$, getting good ocular projection and increasing the airway. ${ }^{4}$

This report aims to show the bennefit of management of severe maxillary asymmetry and unilateral malar hypoplasia using, for the first time, a multi-vectorial bone distraction in the maxillary, integrating 3D planning and a customized polyetheretherketone (PEEK) implant in a sequela for embryonal rhabdomyosarcoma.

\section{CASE DESCRIPTION}

A 16-year-old patient who at ten months of age was diagnosed and treated with chemotherapy and radiotherapy for embryonal rhabdomyosarcoma in the right orbit, and his sequelae are evidenced with severe malar hypoplasia, paranasal deficiency, agenesis of the zygomatic arch, deviated maxilla, and edged towards the right side (Fig 1).

A clinical and computerized tomography (CT) examination made possible a virtual $3 \mathrm{D}$ planning to manage severe maxillary hipoplasia. That information allows the virtual planning movements of the intraoral distractor devices (Fig 2) without anatomical structure interferences. Later, we got a $3 \mathrm{D}$ model print to analyze the desired movement vectors before surgical procedures confirming planned surgical movement and position. The intraoral distractor devices were customized to get

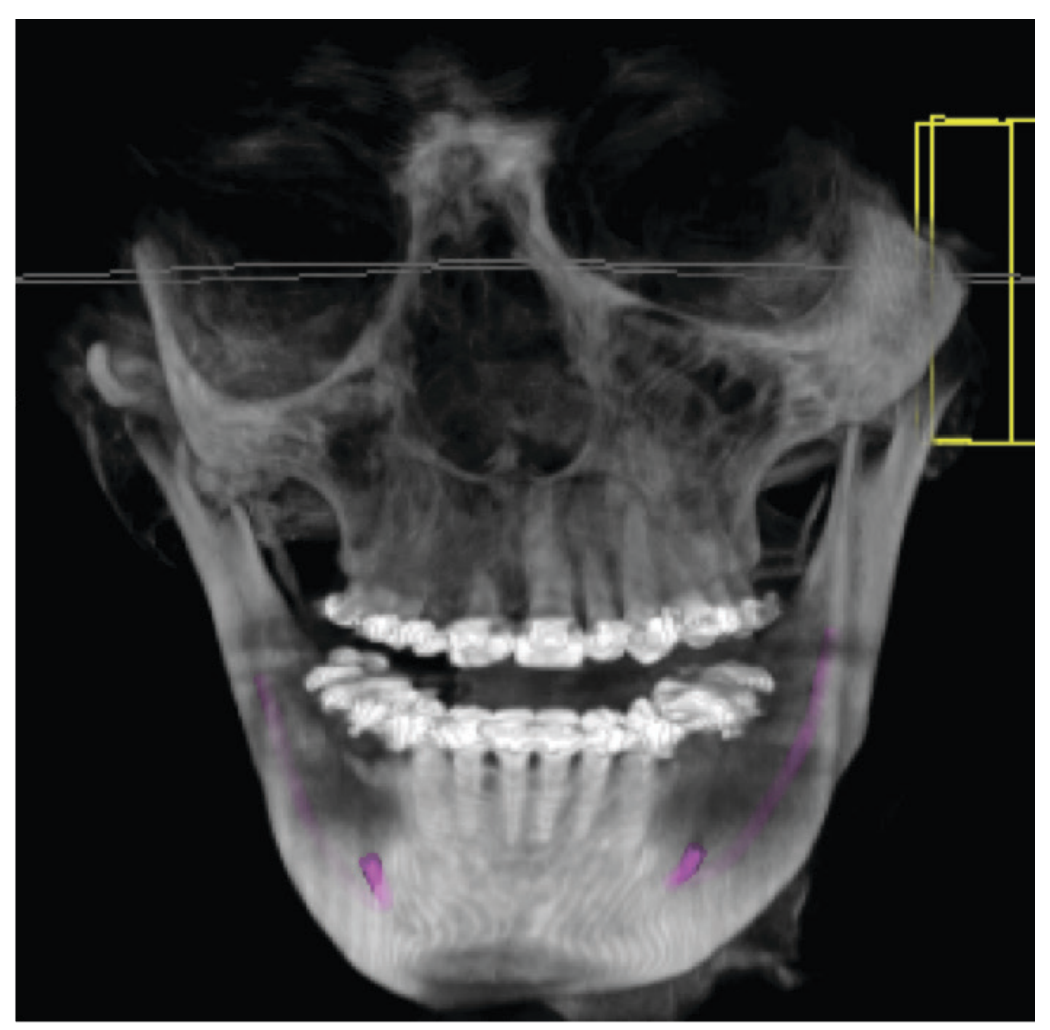

FIGURE 1. Pre-operative computed tomography shows severe right side malar hypoplasia, paranasal deficiency, agenesis of the zygomatic arch, deviated maxilla, and edged towards the right side. 
the compensatory movements as pitch, roll, and yaw to correct the facial asymmetry. With a maxillary vestibular approach from the left first molar to the contralateral molar, it is performed. Le Fort I osteotomy is then performed using a piezoelectric device and 3D splints, which helps avoid damages to supplies and neuronal tissues adjacent to the surgical site. Posteriorly, the distractors devices were placed in the planning 3D position, and then, they were activated by conserving the multi-vectorial distraction. We checked the final surgical position and used absorbable polyglactin suture 4-0. Finally, we started the distraction protocol with five days for the latency period, $1 \mathrm{~mm}$ distraction per day as necessary, and a consolidation phase for eight months (Fig 3).

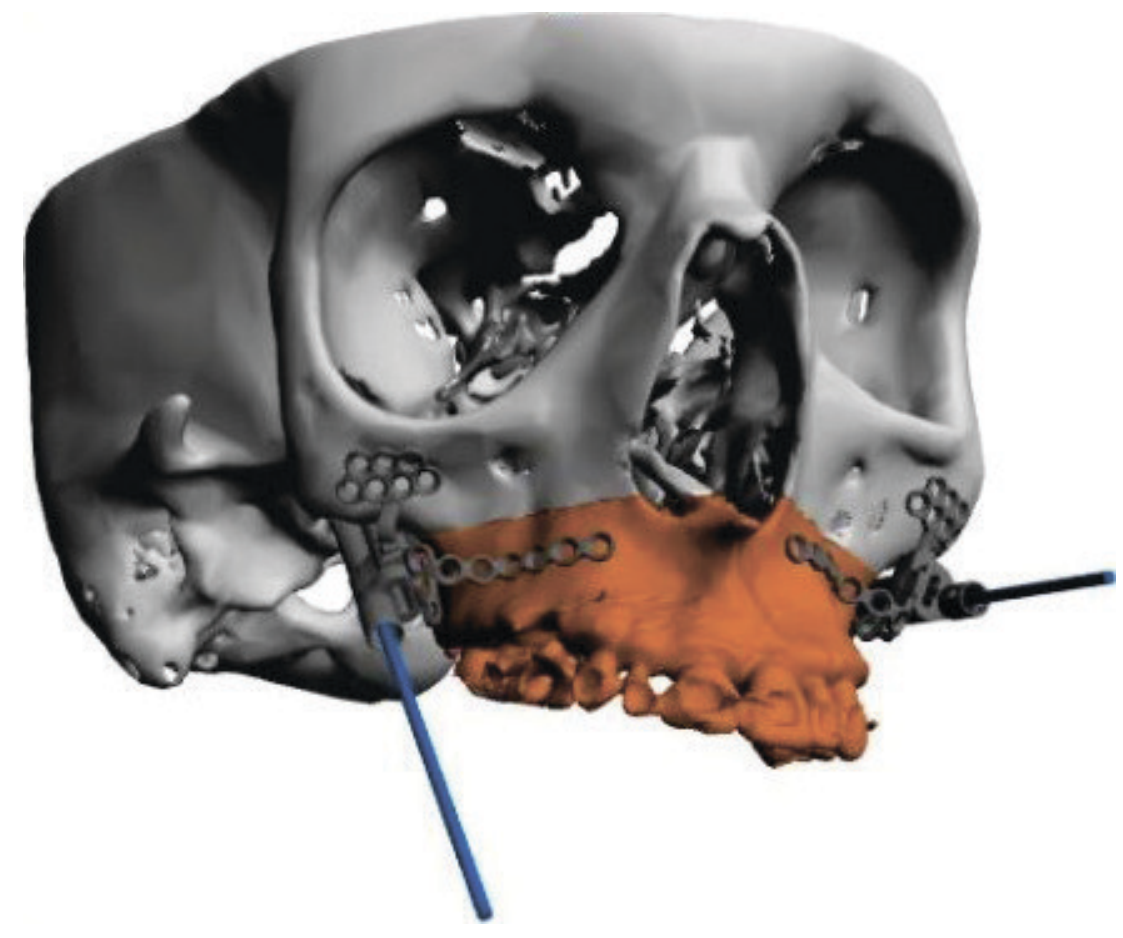

FIGURE 2. Virtual planning of the distractors in different vectors showing vertical and horizontal correction at the same time.
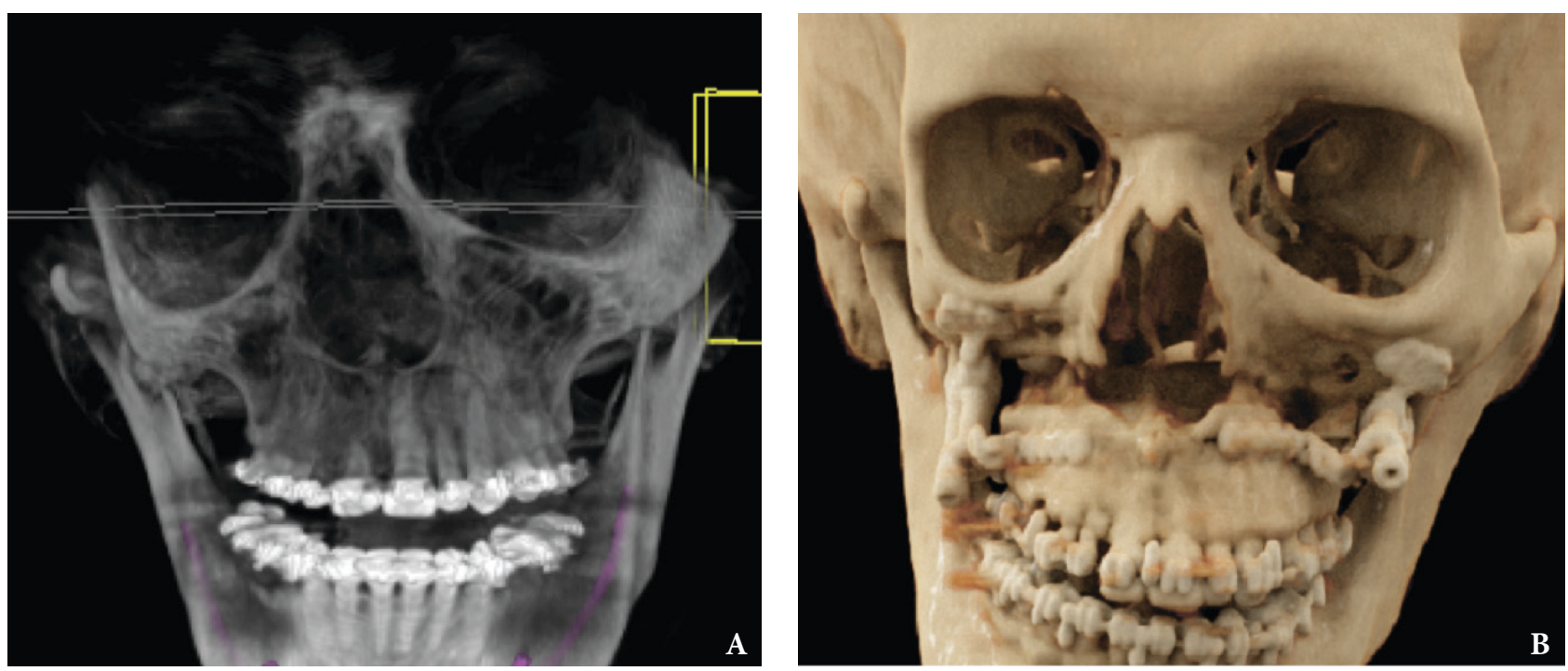

FIGURE 3. CT before $(\mathbf{A})$ and after $(\mathbf{B})$ maxillary distraction. 
Virtual planning of customized PEEK is designed for a better aesthetic result and correction of the malar deficiency (Fig 4). On a second surgical time, distractors are removed throughout of vestibular approach, and PEEK is inserted using the same approach. Finally, the wound is closed by absorbable polyglactin suture 4-0. Changes in maxillary and malar projection were possible with these procedures. However, some limitations can be corrected in the future. Figures 5-7 demonstrate facial photographs before and after surgeries.

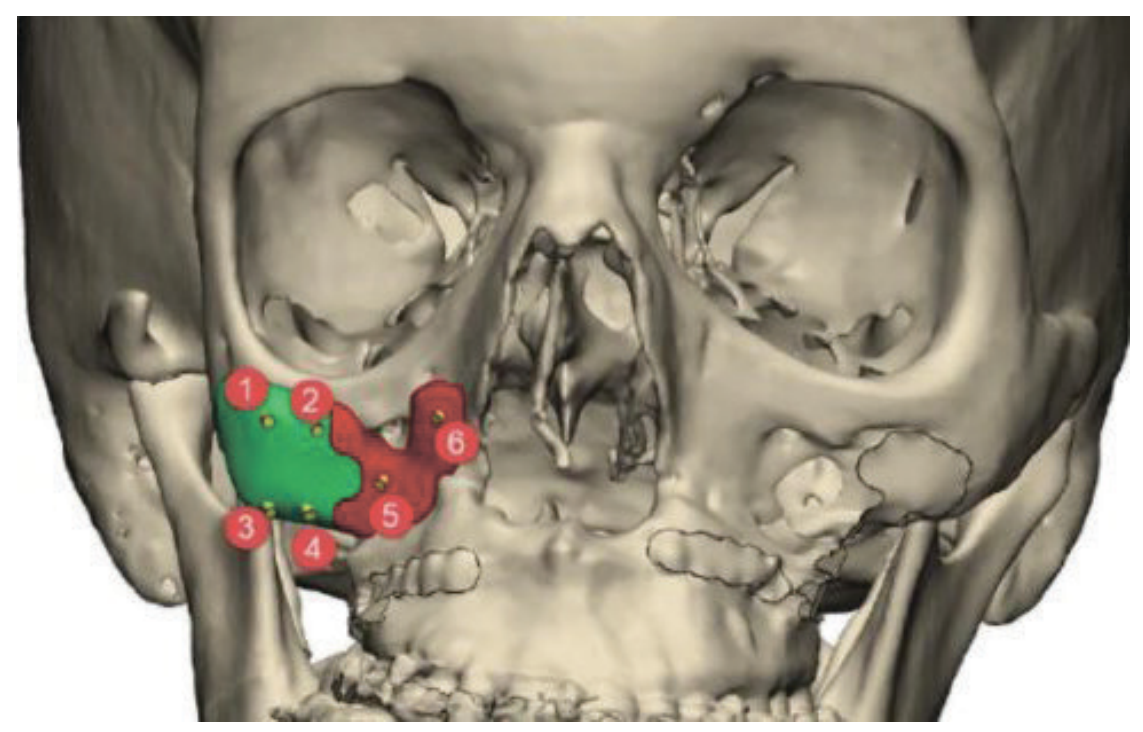

FIGURE 4. Virtual planning of customized PEEK prosthesis for unilateral malar hypoplasia. Numbers (1-6) in red circles indicate the screw holes.
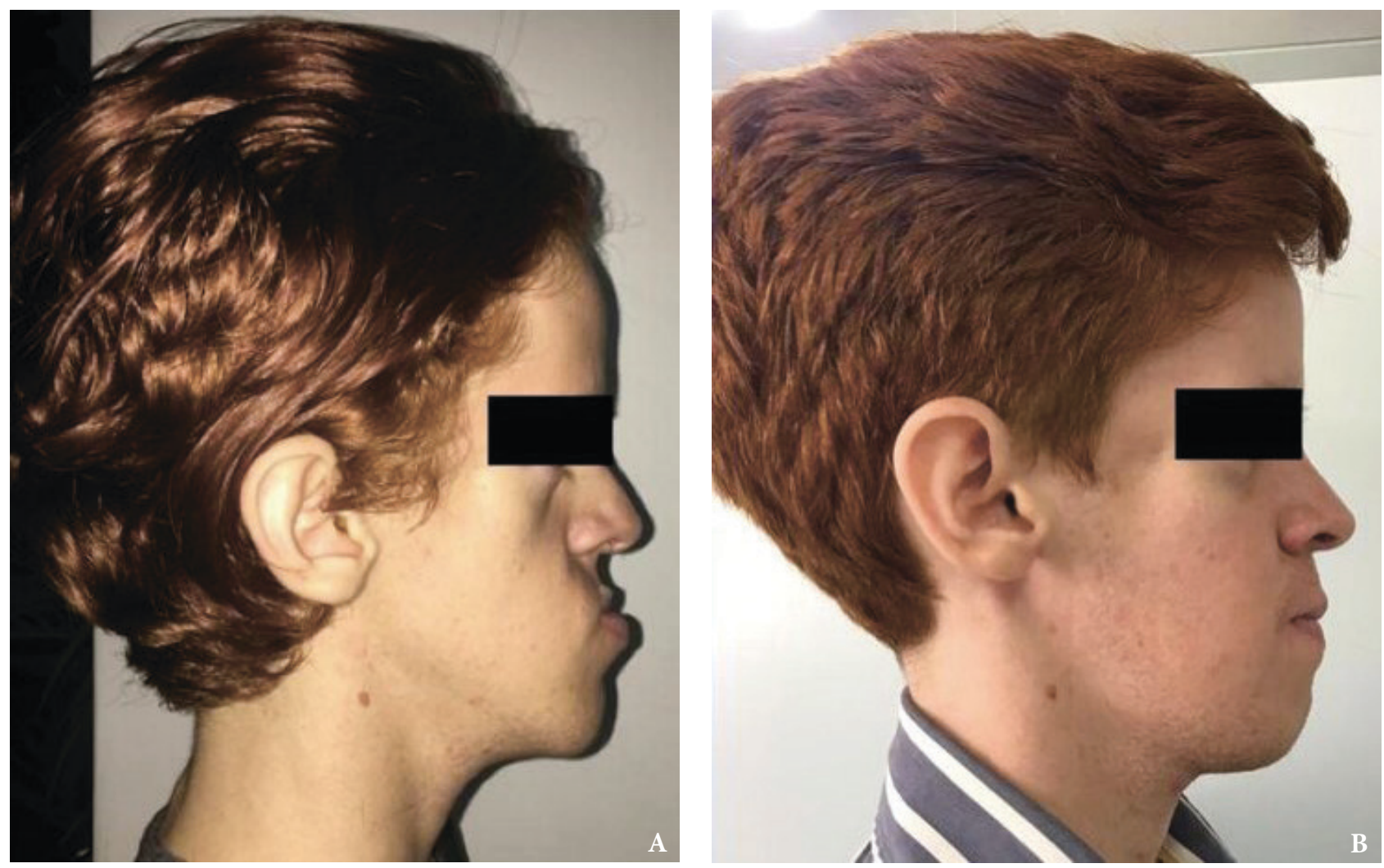

FIGURE 5. Before $(\mathbf{A})$ and after $(\mathbf{B})$ surgeries showing a better malar projection. 

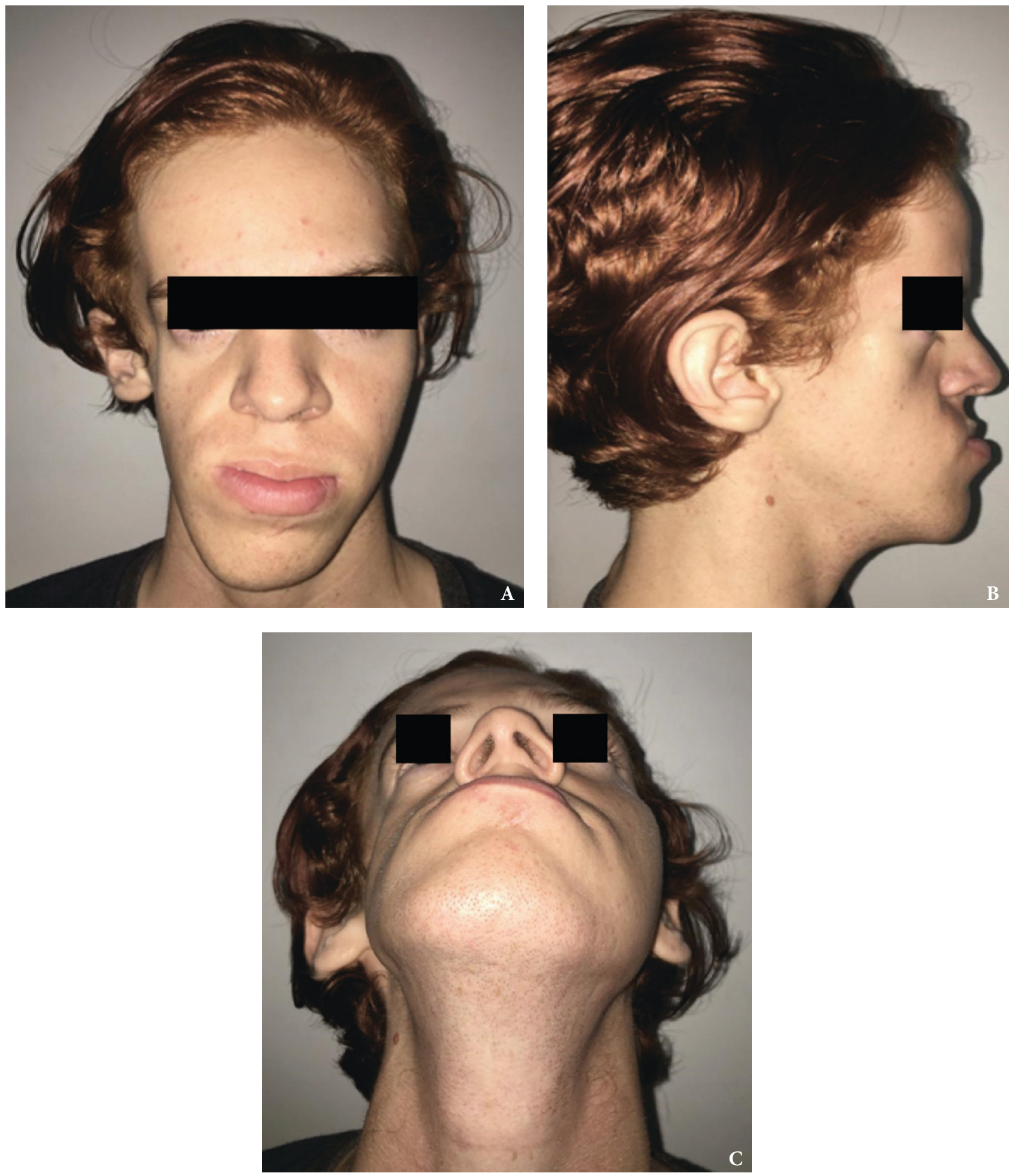

FIGURE 6. Facial photographs taken in frontal view $(\mathbf{A}), 90$ degree lateral view $(\mathbf{B})$, and 45 degree angled view $(\mathbf{C})$ before surgery. 

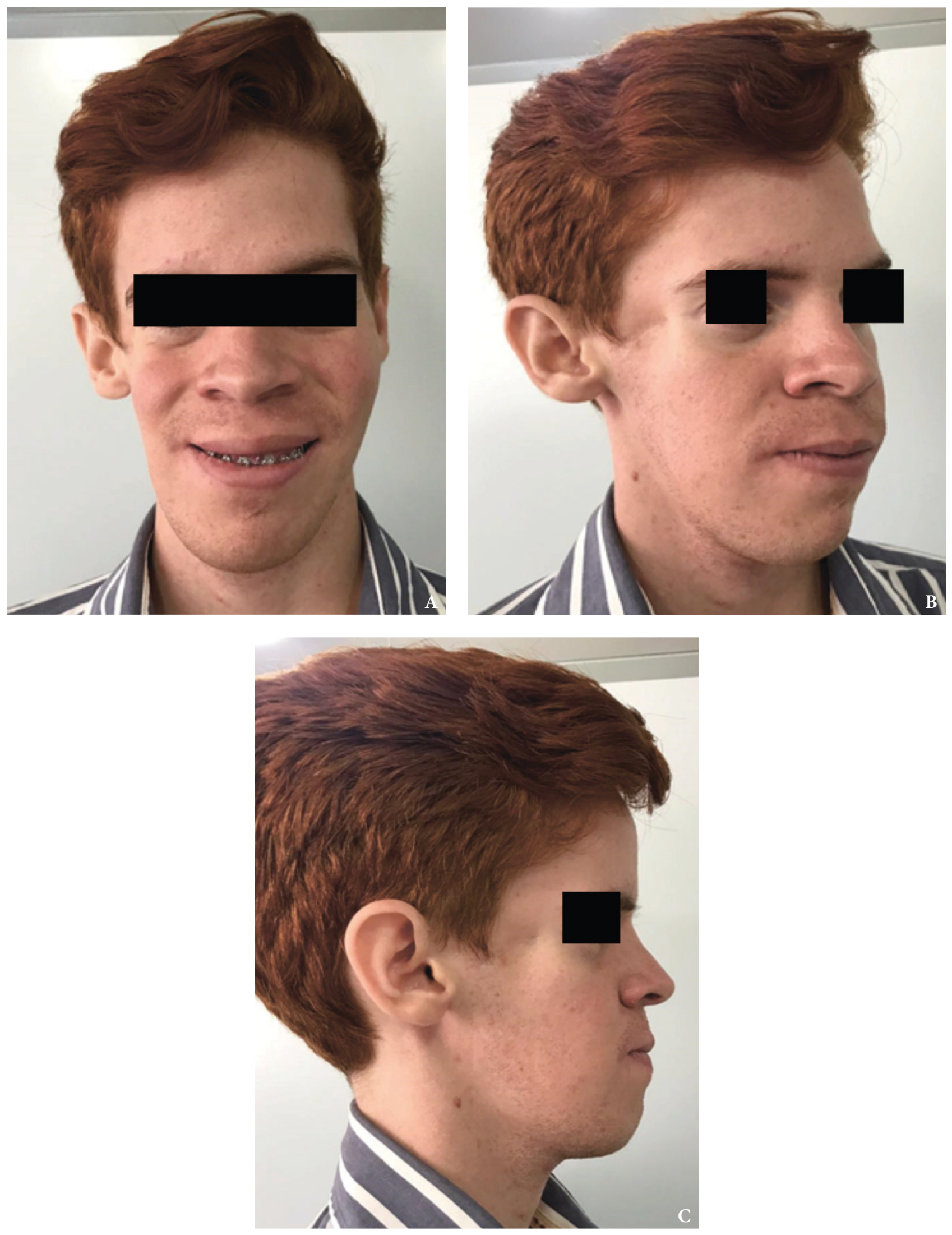

FIGURE 7. Facial photographs taken in frontal view $(\mathbf{A}), 45$ degree angled view $(\mathbf{B}), 90$ degree lateral views $(\mathbf{C})$ after surgery three years later. 


\section{DISCUSSION}

Despite the literature has reported the management for midface severe hypoplasias associated with Pfeiffer, Crouzon, Apert syndromes, the proposed management is to make Le Fort I, II, and III osteotomies depending on clinical findings. ${ }^{5}$ Nowadays, craniofacial surgery is implementing bone distraction with good results. It allows the most significant movements without a requirement of a second procedure to get a donor site bone, which gives us a less invasive surgery and less surgical time, allowing diminished transfusion requirements and hospital cares. ${ }^{6}$ The most relevant difference between orthognathic surgery and osseous distraction is the lesser surgical time. Osteogenic bone distraction decreases surgical time but has more extended postoperative care than orthognathic surgery. The surgeon's challenge is to maintain the vector adequately in postoperative care, but minor changes remain that could modify the results like soft tissue around it.

The virtual planning allowed Gateno et al to implement this technology into surgical procedures such as osseous distraction for the first time in craniofacial surgery in $2003 .{ }^{8}$ However, another study was carried out. Ritto et al sought to compare the precision of virtual planning or conventional models for maxillary positioning. It took into account 30 records of patients undergoing bimaxillary surgery in which there were no statistically significant differences between the two groups for the final result. However, virtual planning did obtain advantages in surgical times and prevent intra-operative complications due to the visualization of the osteotomized segments and the possibility of manipulating them, making planning more user-friendly. ${ }^{9}$ The technology integration for the management of severe facial asymmetries has increased due to the security and trust it provides to the surgeon. Hany et al report a small series of cases integrating virtual planning and $3 \mathrm{D}$ printing of surgical stents to protect relevant anatomical structures and an acceptable therapeutic margin of error in the vectors of mandibular osteogenic distraction controlled from planning. ${ }^{10}$

On the other hand, Bertossi et al demonstrated the advantage of performing an orthognathic surgery with an ultrasonic cut against conventional cutters. They showed that the ultrasonic cutter gives a more proper cut and decreases bleeding risk due to soft tissue protection around osteotomies than conventional ones. ${ }^{11}$ Additionally, we described using customized
PEEK to correct unilateral malar hypoplasia as a virtual 3D planning sequence. This material is similar in physical and mechanical properties to human bone, and also it demonstrated high biocompatibility. Other benefits are low cost and less surgical time, although it needs rigorous pre-surgical virtual planning. ${ }^{12}$

In conclusion, we describe for the first time multivector osteogenic distraction in maxillary asymmetry assisted by $3 \mathrm{D}$ planning in the maxilla to manage severe asymmetries allowing movements in different planes simultaneously. Malar hypoplasia was corrected with maxillary movements and a customized PEEK implant to reduce the midface's asymmetry. Those surgical techniques allowed us to make the most significant movements in the shortest amount of surgical time. Furthermore, ultrasonic devices decrease intraoperative bleeding and postoperative edema, resulting in a more comfortable and safer postoperative period.

\section{FUNDING}

No funding received.

\section{CONFLICT OF INTEREST}

The authors have expressed none conflict of interests.

\section{ETHICAL APPROVAL}

Not necessary.

\section{INFORMED CONSENT}

Informed consent was obtained from the patient.

ACKNOWLEDGMENTS

None.

\section{DECLARATION OF INTEREST STATEMENT}

None of the authors have conflicts of interest.

\section{ROLE OF CO-AUTHORS}

Arango $\mathrm{H}$ (material processing).

Vidal J (editing, writing text).

Moncada JA (writing text, editing).

López JP (concept, design, and writing text). 


\section{REFERENCES}

1. Snyder CC, Levine GA, Swanson HM, Browne EZ Jr. Mandibular lengthening by gradual distraction. Preliminary report. Plast Reconstr Surg 1973;51(5):506-8.

https://doi.org/10.1097/00006534-197305000-00003

2. McCarthy JG, Schreiber J, Karp N, Thorne CH, Grayson BH. Lengthening the human mandible by gradual distraction. Plast Reconstr Surg 1992;89(1):18; discussion 9-10.

3. Rachmiel A, Potparic Z, Jackson IT, Sugihara T, Clayman L, Topf JS, Forté RA. Midface advancement by gradual distraction. Br J Plast Surg 1993;46(3):2017.

https://doi.org/10.1016/0007-1226(93)90169-c

4. Polley JW, Figueroa AA, Charbel FT, Berkowitz R, Reisberg D, Cohen M. Monobloc craniomaxillofacial distraction osteogenesis in a newborn with severe craniofacial synostosis: a preliminary report. $J$ Craniofac Surg 1995;6(5):421-3.

https://doi.org/10.1097/00001665-199509000-00022

5. Katzen JT, McCarthy JG. Syndromes involving craniosynostosis and midface hypoplasia. Otolaryngol Clin North Am 2000;33(6):1257-84. https://doi.org/10.1016/s0030-6665(05)70280-2

6. Swennen G, Schliephake H, Dempf R, Schierle H, Malevez C. Craniofacial distraction osteogenesis: a review of the literature: part 1: clinical studies. Int $J$ Oral Maxillofac Surg 2001;30(2):89-103. https://doi.org/10.1054/ijom.2000.0033

7. Van Sickels JE. Distraction osteogenesis versus orthognathic surgery. Am J Orthod Dentofacial Orthop 2000;118(5):482-4. https://doi.org/10.1067/mod.2000.110517

8. Gateno J, Teichgraeber JF, Xia JJ. Three-dimensional surgical planning for maxillaryand midface distraction osteogenesis. J Craniofac Surg 2003;14(6):833-9. https://doi.org/10.1097/00001665-200311000-00004

9. Ritto FG, Schmitt ARM, Pimentel T, Canellas JV, Medeiros PJ. Comparison of the accuracy of maxillary position between conventional model surgery and virtual surgical planning. Int J Oral Maxillofac Surg 2018;47(2):160-6. https://doi.org/10.1016/j.ijom.2017.08.012

10. Hany HE, El Hadidi YN, Sleem H, Taha M, El Kassaby M. Novel technique and step-by-step construction of a computer-guided stent for mandibular distraction osteogenesis. J Craniofac Surg 2019;30(7):2271-4. https://doi.org/10.1097/SCS.0000000000005614

11. Bertossi D, Lucchese A, Albanese M, Turra M, Faccioni F, Nocini P, Rodriguez $Y$ Baena R. Piezosurgery versus conventional osteotomy in orthognathic surgery: a paradigm shift in treatment. $J$ Craniofac Surg 2013;24(5):1763-6. https://doi.org/10.1097/SCS.0b013e31828f1aa8

12. Zhang J, Tian W, Chen J, Yu J, Zhang J, Chen J. The application of polyetheretherketone (PEEK) implants in cranioplasty. Brain Res Bull 2019;153:143-9. https://doi.org/10.1016/j.brainresbull.2019.08.010 